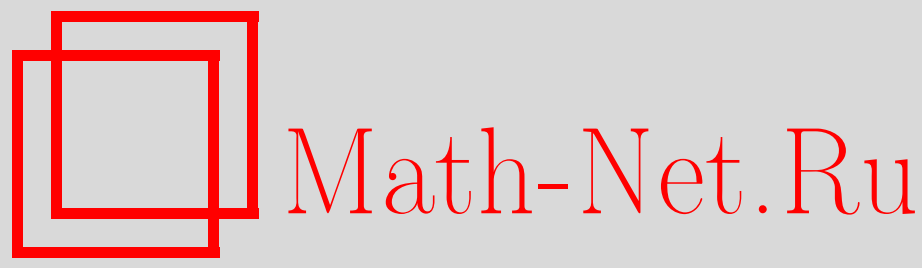

В. Г. Морозов, Г. Рёпке, Двухвременные корреляционные функции в точно решаемой спин-бозонной модели, TMФ, 2011, том 168, номер 3, 482-489

DOI: https://doi.org/10.4213/tmf6695

Использование Общероссийского математического портала Math-Net.Ru подразумевает, что вы прочитали и согласны с пользовательским соглашением http://www.mathnet.ru/rus/agreement

Параметры загрузки:

IP: 54.209 .52 .79

26 апреля 2023 г., 12:52:25

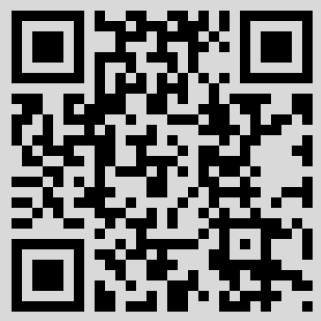




\title{
ДВУХВРЕМЕННЫЕ КОРРЕЛЯЦИОННЫЕ ФУНКЦИИ В ТОЧНО РЕШАЕМОЙ СПИН-БОЗОННОЙ МОДЕЛИ
}

\begin{abstract}
Рассматривается спин-бозонная модель, описывающая процесс дефазировки в открытой квантовой системе. Получены точные выражения для двухвременной спиновой корреляционной функции и функции дефазировки, справедливые при любых значениях констант связи. Показано, что начальные статистические корреляции между динамической системой и термостатом существенно влияют на зависимость функции дефазировки от времени.
\end{abstract}

Ключевые слова: открытые квантовые системы, дефазировка в квантовых системах, спин-бозонные модели.

Посвящается памяти С.В. Тябликова

\section{1. ВВЕДЕНИЕ}

Метод двухвременных функций Грина, разработанный Боголюбовым и Тябликовым [1], обычно используется при изучении спектра квазичастиц и вычислении равновесных величин многочастичных систем. Отметим, однако, что функции Грина и связанные с ними различные корреляционные функции оказываются удобными и для описания неравновесных релаксационных процессов, если отклонение состояния системы от равновесия мало [2], [3]. В настоящей работе мы обсудим применение метода двухвременных корреляционных функций к процессу релаксации в одной из так называемых спин-бозонных моделей [4]-[6].

Любая спин-бозонная модель описывает двухуровневую квантовую систему, взаимодействующую с равновесной макроскопической системой (термостатом), которая представляет собой совокупность осцилляторов. Такого рода модели давно применяются в задачах о квантовом туннелировании в диссипативной среде [4] и в квантовой оптике [7]. В последние двадцать лет интерес к спин-бозонным моделям значительно вырос в связи с тем, что открытая двухуровневая система может играть роль материального носителя единицы квантовой информации - кубита (qubit) -

* Московский государственный институт радиотехники, электроники и автоматики (Технический университет), Москва, Россия. E-mail: vladmorozov45@gmail.com

${ }^{\dagger}$ Institut für Physik, Universität Rostock, Rostock, Germany.

E-mail: gerd.roepke@uni-rostock.de 
в квантовом компьютере (см., например, обзоры [8], [9] и приведенные в них ссылки на оригинальные работы).

Несмотря на формальную простоту спин-бозонных моделей, их динамика оказалась весьма сложной из-за влияния статистических корреляций между двухуровневой системой и термостатом, эффектов памяти и т. д. С фундаментальной и практической точек зрения одним из наиболее интересных процессов в открытых квантовых системах является процесс дефазировки - затухание недиагональных элементов матрицы плотности со временем, в результате чего поведение системы становится квазиклассическим ${ }^{1)}$. В работах [10], [11] была предложена простая спин-бозонная модель, которая описывает процесс дефазировки "в чистом виде", так как в ней отсутствует диссипативный механизм релаксации, приводящий к тепловому равновесию между двухуровневой системой и термостатом. Важным достоинством этой модели является то, что для некоторых начальных состояний матрицу плотности двухуровневой системы удается вычислить точно. Точные решения были найдены и для некоторых других спин-бозонных моделей [4], [6], однако все известные точные решения соответствуют начальному состоянию, в котором отсутствуют статистические корреляции между динамической системой и термостатом.

В настоящей работе на основе метода двухвременных корреляционных функций мы получим точное выражение для матрицы плотности двухуровневой системы в линейном режиме дефазировки с учетом начальных корреляций между динамической системой и термостатом.

\section{2. ОПИСАНИЕ МОДЕЛИ}

Для двухуровневой квантовой системы с базисными состояниями $|0\rangle$ и $|1\rangle$ наиболее удобным является “спиновое” представление, где

$$
|0\rangle=\left(\begin{array}{l}
0 \\
1
\end{array}\right), \quad|1\rangle=\left(\begin{array}{l}
1 \\
0
\end{array}\right),
$$

а все операторы выражаются через матрицы Паули

$$
\sigma_{1}=\left(\begin{array}{cc}
0 & 1 \\
1 & 0
\end{array}\right), \quad \sigma_{2}=\left(\begin{array}{cc}
0 & -i \\
i & 0
\end{array}\right), \quad \sigma_{3}=\left(\begin{array}{cc}
1 & 0 \\
0 & -1
\end{array}\right)
$$

В частности, матрицу плотности такой системы с элементами $\varrho_{i j}(t), i, j=0,1$, можно записать в виде

$$
\varrho(t)=\frac{1}{2}[1+\boldsymbol{\sigma} \cdot \boldsymbol{v}(t)]
$$

где

$$
\boldsymbol{v}(t)=\langle\boldsymbol{\sigma}\rangle^{t} \equiv \operatorname{Tr}\{\boldsymbol{\sigma} \varrho(t)\}
$$

- так называемый вектор Блоха [12], [13].

Гамильтонианы спин-бозонных моделей, которые встречаются в литературе (см., например, работы [4]-[6]), являются частными случаями следующего гамильтониана

1) В англоязычной литературе наряду с термином "дефазировка" (dephasing) часто употребляется термин "decoherence" - потеря когерентности. Буквальный русский перевод последнего термина - декогерентизация [9] - на наш взгляд, не очень удачен. 
(здесь и далее используется система единиц, в которой $\hbar=1$ ):

$$
H=\boldsymbol{\sigma} \cdot \boldsymbol{h}+\sum_{k} \omega_{k} b_{k}^{\dagger} b_{k}+\sum_{i} \sigma_{i} \sum_{k}\left(g_{i k} b_{k}^{\dagger}+g_{i k}^{*} b_{k}\right)
$$

где $\boldsymbol{h}$ - классический вектор, $\omega_{k}$ - частоты мод окружения (термостата), $b_{k}^{\dagger}$ и $b_{k}-$ бозонные операторы рождения и уничтожения для $k$-й моды. Величины $g_{i k}-$ константы связи, характеризующие взаимодействие двухуровневой системы с термостатом.

Мы рассмотрим вариант спин-бозонной модели (2.5) с гамильтонианом [10], [11]

$$
H=\frac{\omega_{0}}{2} \sigma_{3}+\sum_{k} \omega_{k} b_{k}^{\dagger} b_{k}+\sigma_{3} \sum_{k}\left(g_{k} b_{k}^{\dagger}+g_{k}^{*} b_{k}\right)
$$

где $\omega_{0}$ - разность энергий возбужденного $(|1\rangle)$ и основного $(|0\rangle)$ состояний. Следует отметить, что модель (2.6) неэргодическал, так как оператор $\sigma_{3}$ коммутирует с гамильтонианом и, следовательно, среднее значение $\left\langle\sigma_{3}\right\rangle^{t}$ (разность заселенностей уровней) является интегралом движения.

\section{3. ЛИНЕЙНЫЙ РЕЖИМ ДЕФАЗИРОВКИ}

В модели (2.6) приведенная матрица плотности $\varrho(t)$ двухуровневой системы $(S)$ точно вычисляется [6], [10], [11], если в начальный момент времени $t=0$ отсутствуют корреляции между системой и термостатом $(B)$, т. е. начальная матрица плотности $\varrho_{S B}(0)$ составной системы имеет вид прямого произведения:

$$
\varrho_{S B}(0)=\varrho(0) \otimes \varrho_{B}, \quad \varrho_{B}=\frac{e^{-\beta H_{B}}}{Z_{B}},
$$

где $\beta=1 / k_{\mathrm{B}} T, H_{B}$ и $Z_{B}$ - гамильтониан и статистическая сумма термостата. В более реалистичной постановке задачи следует учесть начальные статистические корреляции, которые могут существенно влиять на процесс дефазировки.

С помощью соотношения (2.3) легко проверить, что недиагональные элементы матрицы плотности подсистемы $S$ можно записать в виде

$$
\varrho_{01}(t)=\left\langle\sigma_{+}\right\rangle^{t}, \quad \varrho_{10}(t)=\left\langle\sigma_{-}\right\rangle^{t},
$$

где введены операторы $\sigma_{ \pm}=\left(\sigma_{1} \pm i \sigma_{2}\right) / 2$. Таким образом, поскольку $\varrho_{10}(t)=\varrho_{01}^{*}(t)$, процесс дефазировки полностью описывается зависимостью среднего $\left\langle\sigma_{+}\right\rangle^{t}$ от времени.

Если подсистемы $S$ и $B$ находятся в равновесии друг с другом, то из структуры гамильтониана (2.6) следует, что $\left\langle\sigma_{ \pm}\right\rangle=0$. Мы рассмотрим линейный процесс дефазировки, когда неравновесные средние $\left\langle\sigma_{ \pm}\right\rangle^{t}$ достаточны малы и могут быть вычислены в рамках теории линейной реакции [3]. Предположим, что начальное коррелированное состояние составной системы с $\left\langle\sigma_{ \pm}\right\rangle \neq 0$ описывается матрицей плотности

$$
\varrho_{S B}(0)=\frac{e^{-\beta\left(H-f^{*} \sigma_{+}-f \sigma_{-}\right)}}{Z_{S B}},
$$


где $Z_{S B}$ - нормировочная постоянная, а вспомогательные "внешние поля" $f$ и $f^{*}$ находятся из условий

$$
\left.\left\langle\sigma_{ \pm}\right\rangle\right|_{t=0}=\operatorname{Tr}\left\{\varrho_{S B}(0) \sigma_{ \pm}\right\} .
$$

В линейном приближении по $f$ и $f^{*}$ имеем [3]

$$
\left.\left\langle\sigma_{+}\right\rangle^{t}\right|_{t=0}=\beta\left(\sigma_{+}, \sigma_{-}\right) f .
$$

Равновесная корреляционная функция $\left(\sigma_{+}, \sigma_{-}\right)$является частным случаем двухвременной корреляционной функции, которая определяется для произвольных гейзенберговских операторов $A(t)$ и $B\left(t^{\prime}\right)$ выражением

$$
\left(A(t), B\left(t^{\prime}\right)\right)=\int_{0}^{1} d x\left\langle\Delta A(t-i \beta x) \Delta B\left(t^{\prime}\right)\right\rangle,
$$

где $\Delta A(t)=A(t)-\langle A\rangle$ и $\Delta B\left(t^{\prime}\right)=B\left(t^{\prime}\right)-\langle B\rangle$. Усреднение в формуле (3.6) производится с равновесной матрицей плотности составной системы

$$
\varrho_{S B}^{(\mathrm{eq})}=\frac{e^{-\beta H}}{Z_{S B}^{(\mathrm{eq})}} .
$$

В любой момент времени $t>0$ линейное приближение для среднего $\left\langle\sigma_{+}\right\rangle^{t}$ имеет вид [3]

$$
\left\langle\sigma_{+}\right\rangle^{t}=\beta\left(\sigma_{+}(t), \sigma_{-}\right) f .
$$

Исключая вспомогательное поле $f$ в соотношениях (3.5) и (3.8), получаем

$$
\left\langle\sigma_{+}\right\rangle^{t}=\left.\frac{\left(\sigma_{+}(t), \sigma_{-}\right)}{\left(\sigma_{+}, \sigma_{-}\right)} \cdot\left\langle\sigma_{+}\right\rangle^{t}\right|_{t=0} .
$$

Таким образом, линейный режим дефазировки в двухуровневой системе $S$ полностью описывается корреляционной функцией операторов $\sigma_{+}(t)$ и $\sigma_{-}$в равновесном состоянии составной системы.

\section{4. СПИНОВАЯ КОРРЕЛЯЦИОННАЯ ФУНКЦИЯ}

В этом разделе мы получим точное выражение для временно́й корреляционной функции $\left(\sigma_{+}(t), \sigma_{-}\right)$в модели $(2.6)$.

Введем унитарный оператор

$$
U=\exp \left(\frac{1}{2} \sigma_{3} K\right)
$$

где

$$
K=2 \sum_{k} \frac{1}{\omega_{k}}\left(g_{k} b_{k}^{\dagger}-g_{k}^{*} b_{k}\right)
$$

- оператор, действующий только на состояния термостата. Для произвольного оператора $A$ введем преобразованный оператор

$$
\widetilde{A}=U A U^{-1} \text {. }
$$


Используя определение (4.1), получаем выражения для преобразованных бозонных операторов:

$$
\tilde{b}_{k}=b_{k}-\frac{g_{k}}{\omega_{k}} \sigma_{3}, \quad \tilde{b}_{k}^{\dagger}=b_{k}^{\dagger}-\frac{g_{k}^{*}}{\omega_{k}} \sigma_{3} .
$$

Очевидно, что $\widetilde{\sigma}_{3}=\sigma_{3}$, поэтому с помощью соотношений (4.4) легко находится преобразованный гамильтониан составной системы (2.6):

$$
\widetilde{H}=-\sum_{k} \frac{\left|g_{k}\right|^{2}}{\omega_{k}}+\frac{\omega_{0}}{2} \sigma_{3}+\sum_{k} \omega_{k} b_{k}^{\dagger} b_{k} \equiv E_{0}+H_{S}+H_{B}
$$

Заметим, что преобразованный гамильтониан описывает невзаимодействующие подсистемъ.

Рассмотрим теперь корреляционную функцию $\left(A(t), B\left(t^{\prime}\right)\right)$, где $A$ и $B$ - некоторые операторы. С помощью соотношений (4.3) и (4.5) легко проверить, что

$$
\left(A(t), B\left(t^{\prime}\right)\right)=\left(\widetilde{A}(t), \widetilde{B}\left(t^{\prime}\right)\right)_{0},
$$

причем корреляционная функция в правой части вычисляется с матрицей плотности

$$
\varrho_{0} \equiv \widetilde{\varrho}_{S B}^{(\mathrm{eq})}=\varrho_{S} \otimes \varrho_{B}
$$

где

$$
\varrho_{S}=\frac{e^{-\beta H_{S}}}{Z_{S}}, \quad \varrho_{B}=\frac{e^{-\beta H_{B}}}{Z_{B}},
$$

а зависимость от времени преобразованного оператора Гейзенберга определяется соотношением

$$
\widetilde{A}(t)=e^{i t\left(H_{S}+H_{B}\right)} \widetilde{A} e^{-i t\left(H_{S}+H_{B}\right)} .
$$

Применим формулу (4.6) к равновесной корреляционной функции $\left(\sigma_{+}(t), \sigma_{-}\right)$. Предварительно с помощью выражения (4.1) найдем правила преобразования для спиновых операторов: $\widetilde{\sigma}_{ \pm}=e^{ \pm K} \sigma_{ \pm}$. После этого формула (4.6) дает

$$
\left(\sigma_{+}(t), \sigma_{-}\right)=\int_{0}^{1} d x\left\langle\sigma_{+}(t-i \beta x) \sigma_{-}\right\rangle_{S}\left\langle e^{K(t-i \beta x)} e^{-K}\right\rangle_{B},
$$

где средние значения $\langle\cdot\rangle_{S}$ и $\langle\cdot\rangle_{B}$ находятся соответственно с матрицами плотности $\varrho_{S}$ и $\varrho_{B}$, а представление Гейзенберга для операторов определяется гамильтонианами подсистем $H_{S}$ и $H_{B}$.

Средние значения в правой части соотношения (4.10) точно вычисляются, так как для произвольного комплексного $z$ имеем

$$
\sigma_{+}(z)=e^{i z \omega_{0}} \sigma_{+}, \quad b_{k}(z)=e^{-i z \omega_{k}} b_{k}, \quad b_{k}^{\dagger}(z)=e^{i z \omega_{k}} b_{k}^{\dagger},
$$

а оператор (4.2) линеен по бозонным операторам $b_{k}$ и $b_{k}^{\dagger}$. Опуская элементарные преобразования, приведем окончательное выражение для спиновой корреляционной функции:

$$
\left(\sigma_{+}(t), \sigma_{-}\right)=\frac{e^{i \omega_{0} t}}{e^{\beta \omega_{0}}+1} \int_{0}^{1} d x e^{\beta \omega_{0} x-R(t-i \beta x)},
$$


где

$$
R(z)=\sum_{k} \frac{4\left|g_{k}\right|^{2}}{\omega_{k}^{2}}\left[\left(1-\cos \left(\omega_{k} z\right)\right) \operatorname{cth} \frac{\beta \omega_{k}}{2}+i \sin \left(\omega_{k} z\right)\right]
$$

Спектр мод термостата $\omega_{k}$ практически непрерывен. Поэтому, вводя спектральную плотность термостата $J(\omega)$ с помощью равенства

$$
\sum_{k} 4\left|g_{k}\right|^{2} f\left(\omega_{k}\right)=\int_{0}^{\infty} d \omega J(\omega) f(\omega)
$$

мы можем записать формулу (4.13) в виде

$$
R(z)=\int_{0}^{\infty} d \omega \frac{J(\omega)}{\omega^{2}}\left[(1-\cos (\omega z)) \operatorname{cth} \frac{\beta \omega}{2}+i \sin (\omega z)\right] .
$$

Полагая здесь $z=t-i \beta x$, из (4.12) получаем выражение

$$
\left(\sigma_{+}(t), \sigma_{-}\right)=\frac{e^{i \omega_{0} t}}{e^{\beta \omega_{0}}+1} \int_{0}^{1} d x e^{\beta \omega_{0} x-F(t, x)+i \Phi(t, x)}
$$

с действительными функциями

$$
\begin{aligned}
F(t, x)= & \int_{0}^{\infty} d \omega \frac{J(\omega)}{\omega^{2}}\left[1-e^{-\omega \beta x}-\frac{4 \operatorname{sh}^{2}(\omega \beta x / 2)}{e^{\beta \omega}-1}\right]+ \\
& +\int_{0}^{\infty} d \omega \frac{J(\omega)}{\omega^{2}}\left[\frac{2 \operatorname{ch}(\omega \beta x)}{e^{\beta \omega}-1}+e^{-\omega \beta x}\right](1-\cos (\omega t)), \\
\Phi(t, x)= & \int_{0}^{\infty} d \omega \frac{J(\omega)}{\omega^{2}}\left[\frac{2 \operatorname{sh}(\omega \beta x)}{e^{\beta \omega}-1}-e^{-\omega \beta x}\right] \sin (\omega t) .
\end{aligned}
$$

Подчеркнем, что в рамках модели (2.6) результат (4.16) является точным. Чтобы найти явную зависимость корреляционной функции от времени, требуется задать конкретное выражение для спектральной плотности термостата $J(\omega)$.

\section{5. ДЕФАЗИРОВКА В ДВУХУРОВНЕВОЙ СИСТЕМЕ}

Напомним, что согласно соотношению (3.9) спиновая корреляционная функция $\left(\sigma_{+}(t), \sigma_{-}\right)$описывает процесс дефазировки в подсистеме $S$. Соотношение (3.9) удобно представить в форме

$$
\left\langle\sigma_{+}\right\rangle^{t}=\left.e^{-\Gamma(t)} e^{i\left[\omega_{0} t+\chi(t)\right]} \cdot\left\langle\sigma_{+}\right\rangle^{t}\right|_{t=0},
$$

где $\Gamma(t)$ называется функцией дефазировки (decoherence function) [6]. Выражения для $\Gamma(t)$ и зависящего от времени фазового сдвига $\chi(t)$ легко найти с помощью формулы (4.16):

$$
\Gamma(t)=\frac{1}{2} \ln \left[\frac{I_{0}^{2}}{I_{\mathrm{c}}^{2}(t)+I_{\mathrm{s}}^{2}(t)}\right], \quad \chi(t)=\operatorname{arctg} \frac{I_{\mathrm{s}}(t)}{I_{\mathrm{c}}(t)},
$$


где

$$
\begin{aligned}
I_{0} & =\int_{0}^{1} d x e^{\beta \omega_{0} x} e^{-F(0, x)}, \\
I_{\mathrm{c}}(t) & =\int_{0}^{1} d x e^{\beta \omega_{0} x} e^{-F(t, x)} \cos [\Phi(t, x)], \\
I_{\mathrm{s}}(t) & =\int_{0}^{1} d x e^{\beta \omega_{0} x} e^{-F(t, x)} \sin [\Phi(t, x)] .
\end{aligned}
$$

Ранее функция дефазировки $\Gamma(t)$ в спин-бозонной модели (2.6) вычислялась для начального состояния (3.1), в котором отсутствуют статистические корреляции между подсистемой $S$ и термостатом [6], [10], [11], а для спектральной плотности термостата $J(\omega)$ использовалось модельное выражение

$$
J(\omega)=\lambda \omega e^{-\omega / \Omega},
$$

где $\lambda$ - безразмерная константа связи, а $\Omega$ - некоторая "обрезающая" частота для спектра мод термостата. В этом случае, если $\beta \Omega \gg 1$, функцию дефазировки удается найти в явном виде (см., например, [6]):

$$
\Gamma(t)=\frac{\lambda}{2} \ln \left(1+\Omega^{2} t^{2}\right)+\lambda \ln \left[\frac{\operatorname{sh}\left(t / \tau_{\mathrm{B}}\right)}{t / \tau_{\mathrm{B}}}\right] .
$$

Величина

$$
\tau_{\mathrm{B}}=\frac{\beta}{\pi} \equiv \frac{\hbar}{\pi k_{\mathrm{B}} T}
$$

- так называемое "тепловое время корреляции". Первый член в формуле (5.5) соответствует "вакуумным" квантовым флуктуациям в термостате и является основным

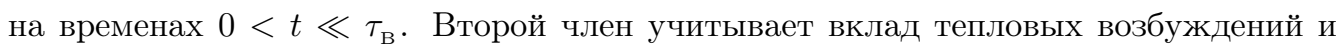
определяет поведение функции дефазировки при $t \gtrsim \tau_{\mathrm{B}}$.

Для коррелированного начального состояния зависимость функции дефазировки от времени является более сложной. Хотя явный вид $\Gamma(t)$ (см. формулу (5.2)) можно найти только численно, легко заметить некоторые важные качественные различия между процессами дефазировки в двух указанных случаях. Во-первых, для коррелированного начального состояния невозможно разделить в $\Gamma(t)$ вклады "вакуумных" флуктуаций и тепловых возбуждений термостата. Во-вторых, в этом случае поведение функции дефазировки зависит от значения параметра $\beta \omega_{0}$, который вообще не входит в выражение (5.5). Наконец, начальные корреляции между двухуровневой подсистемой и термостатом приводят к появлению зависящего от времени фазового сдвига $\chi(t)$ в недиагональных элементах матрицы плотности подсистемы. Наличие этого фазового сдвига может существенно влиять, например, на свойства квантовых регистров, состоящих из нескольких двухуровневых систем кубитов [11], так как различие фазовых сдвигов $\chi(t)$ в кубитах является дополнительным механизмом дефазировки всего регистра.

\section{6. ЗАКЛЮЧЕНИЕ}

Мы рассмотрели пример вычисления двухвременной корреляционной функции непосредственно в $t$-представлении без использования обычного подхода - построения цепочки уравнений для корреляционных функций высших порядков, зависящих 
от частоты. Ключевым моментом, позволившим получить точное выражение для корреляционной функции в модели (2.6), является применение унитарного преобразования (4.3) с оператором (4.1), в результате чего задача свелась к вычислению средних для двух невзаимодействующих подсистем. В более общих спин-бозонных моделях (2.5) указанное унитарное преобразование не позволяет полностью исключить оператор взаимодействия в гамильтониане, однако оно может оказаться полезным для анализа тех моделей, где основное взаимодействие между динамической системой и термостатом описывается таким же оператором, как и в гамильтониане (2.6) (см., например, статью [4]). В подобных случаях преобразование корреляционных функций, аналогичное (4.6), упрощает применение теории возмущений по "остаточному" слабому взаимодействию. Мы планируем рассмотреть этот вопрос более подробно в отдельной работе.

Благодарности. Работа поддержана грантом Deutsche Forschungsgemeinschaft и Sonderforschungsbereich - Collective Research Center 652.

\section{Список литературы}

[1] Н. Н. Боголюбов, С. В. Тябликов, Докл. АН СССР, 126:1 (1959), 53-56.

[2] Д. Н. Зубарев, Неравновесная статистическая термодинамика, Наука, М., 1971.

[3] Д. Н. Зубарев, В. Г. Морозов, Г. Рёпке, Статистическая механика неравновесных проиессов, Физматлит, М., 2002.

[4] A. J. Leggett, S. Chakravarty, A. T. Dorsey, M. P. A. Fisher, A. Garg, W. Zwerger, Rev. Mod. Phys., 59:1 (1987), 1-85; Erratum 67 (1995), 725-726.

[5] U. Weiss, Quantum Dissipative Systems, Ser. Modern Condens. Matter Phys. 10, World Scientific, Singapore, 1999.

[6] H.-P. Breuer, F. Petruccione, The Theory of Open Quantum Systems, Oxford Univ. Press, Oxford, 2002.

[7] M. O. Scully, M. S. Zubairy, Quantum Optics, Cambridge Univ. Press, Cambridge, 1997.

[8] D. Bouwmeester, A. Ekert, A. Zeilinger (eds.), The Physics of Quantum Information. Quantum Cryptography, Quantum Teleportation, Quantum computation, Springer, Berlin, 2000.

[9] К. А. Валиев, УФН, 175:1 (2005), 3-39.

[10] W. G. Unruh, Phys. Rev. A, 51:2 (1995), 992-997.

[11] G. M. Palma, K.-A. Suominen, A. K. Ekert, Proc. R. Soc. Lond. A, 452:1946 (1996), $567-584$.

[12] L. Allen, J. H. Eberly, Optical Resonance and Two-level Atoms, Dover, New York, 1975.

[13] C. Cohen-Tannoudji, B. Diu, F. Laloë, Quantum Mechanics, Wiley, New York, 1977.

Поступила в редакцию 8.03.2011 